\title{
One-pot multi-component synthesis of 1,4-dihydropyridine derivatives in biocompatible deep eutectic solvents
}

\author{
SUHAS PEDNEKAR, RAHUL BHALERAO and NITIN GHADGE* \\ Organic Chemistry Research Laboratory, Department of Chemistry, Ramnarain Ruia College, \\ Matunga, Mumbai 400 019, India \\ e-mail: nitin.ghadge3@gmail.com
}

MS received 24 May 2012; revised 29 September 2012; accepted 6 November 2012

\begin{abstract}
An efficient protocol for the synthesis of differently substituted 1, 4-dihydropyridines in deep eutectic solvents under solvent-free conditions is reported here. Excellent yields of the resultant products have been obtained. Recyclability studies have also been performed for deep eutectic solvents with very little loss in activity up to five recycles.
\end{abstract}

Keywords. Green chemistry; one-pot synthesis; 1,4-dihydropyridines; deep eutectic solvents; recyclability.

\section{Introduction}

1,4-Dihydropyridines (1,4-DHPs) are an important class of bioactive molecules, well-known for their role as calcium channel modulators and used extensively for the treatment of hypertension. ${ }^{1-3}$ The derivatives of 1,4-DHP have shown a variety of biological activities such as vasodilator, bronchodilator, antitumour, hepatoprotective and geroprotective activities. ${ }^{4,5}$ Commercial drugs such as Nifedipine which are a prototype of the 1,4-DHP structure have been used extensively in both antianginal and antihypertensive treatment. ${ }^{6}$

One-pot multi-component reactions are of increasing academic and ecological interest due to the possibility of achieving high synthetic efficiency and reaction design. ${ }^{7}$ The classical method for synthesis of 1,4DHPs involves one-pot condensation of three or more components under relatively harsh conditions using ammonia as the nitrogen source. ${ }^{8}$ Drastic reduction in reaction time and improved reactivity of the synthetic procedures have been brought about by the application of microwaves. ${ }^{9}$ Although, impressive levels of activity could be achieved, large-scale synthesis is a problem. Similarly, Lewis acid catalysts such as $\mathrm{Yb}(\mathrm{OTf})_{3}$ have been shown to catalyse the reactions at ambient temperature. ${ }^{10}$

*For correspondence
Recently, Zhao-Qin Jiang et al. reported the synthesis of 1,4-DHP derivatives in ionic liquids. ${ }^{11}$ Ionic liquids have attracted much attention during the last decade in the context of green chemistry due to their low vapour pressure, high thermal stability and their solvation properties which make them such unique solvents in synthesis. ${ }^{12}$ However, serious limitations have emerged for the industrial scale application of ionic liquids such as high cost, ${ }^{13}$ environmental toxicity ${ }^{14}$ and demand for high purity which could otherwise lead to change in the physical properties of ionic liquids. ${ }^{15,16}$ The development of alternative solvents from components that are inexpensive, non-toxic towards the environment and are biodegradable is therefore highly desirable to overcome these drawbacks.

One such alternative approach could be the use of deep eutectic solvents (DES) which were first synthesized by Abbott et al. ${ }^{17}$ and since then found wide scope in an array of biological applications due to their biodegradable properties. Most common among them are biological transformations such as hydrolasecatalysed biotransformation ${ }^{18}$ and extraction of glycerol from biodiesel into a eutectic-based solvent. ${ }^{19}$ Although attractive, deep eutectic solvents have seldom been used for organic transformations. ${ }^{20} \mathrm{We}$ therefore thought it would be interesting to try the application of DES in one-pot synthesis of 1,4-DHPs and it worked very efficiently. A mixture of substituted aldehyde 2a-n, dimedone $\mathbf{3}$, ethyl acetoacetate $\mathbf{4}$ and ammonium acetate $\mathbf{5}$ in DES 1a on heating resulted in the formation of 1,4-DHPs $\mathbf{6 a - n}$. 


\section{Experimental}

\subsection{Materials}

All common reagents and solvents were used as obtained from commercial supplies without further purification.

\subsection{Apparatus}

All melting points were determined in open capillaries using Gallen Kamp melting point apparatus and are uncorrected. All ${ }^{1} \mathrm{H}$ NMR (nuclear magnetic resonance) spectra were recorded in $\mathrm{CDCl}_{3}$ (chemical shift in delta) using Bruker AV 200 spectrophotometer (300 MHz).

\subsection{Representative procedure for the preparation of deep eutectic solvent $\mathbf{1 a}$}

Choline chloride A $(2.8 \mathrm{~g}, 20 \mathrm{mmol})$ and urea $\mathbf{B}$ (a) $(2.4 \mathrm{~g}, 40 \mathrm{mmol})$ were placed in a round bottomed flask and heated to $60^{\circ} \mathrm{C}$, until liquid began to form. After $10 \mathrm{~min}$, a homogenous colourless liquid was formed which was used directly for the reactions.

\subsection{Representative procedure for the synthesis of 1,4-DHP derivatives $\mathbf{6 a}-\mathbf{n}$}

2a-n (1.0 mmol), 3 (0.140 g, $1.0 \mathrm{mmol}), 4$ (0.130 g, $1.0 \mathrm{mmol}), \mathbf{5}(0.115 \mathrm{~g}, 1.5 \mathrm{mmol})$ and DES 1a $(2.0 \mathrm{~mL})$ were successively charged into a Schlenk tube equipped with a magnetic stirrer. Then the reaction was stirred at $60^{\circ} \mathrm{C}$ for $20 \mathrm{~min}$ and a solid product gradually formed. After the completion of reaction as indicated by thinlayer chromatography (TLC), water was added, the reaction mass was stirred and the mixture was filtered. The crude product obtained was purified by recrystallization using absolute alcohol. Further, water was removed under vacuum at $80^{\circ} \mathrm{C}$ leaving behind DES which was used up to 5 recycles. A detailed account of the recycle studies is mentioned in results and discussion.

\subsection{Spectroscopic data of selected compounds}

2.5a Ethyl-1,4,5,6,7,8-hexahydro-2,7,7-trimethyl-5oxo-4-phenylquinoline-3-carboxylate (6a): M.p: 203-204 ${ }^{\circ} \mathrm{C}$. IR $\left(\mathrm{KBr}\right.$ in $\left.\mathrm{cm}^{-1}\right)$ 3233, 3210, 3080, 1696, 1602, 1059, 692, ${ }^{1} \mathrm{H} \mathrm{NMR}\left(\mathrm{CDCl}_{3}, 300 \mathrm{MHz}\right): \delta 0.94$ (s, 3H), $1.07(\mathrm{~s}, 3 \mathrm{H}), 1.21(\mathrm{t}, J=7.1 \mathrm{~Hz}, 3 \mathrm{H}), 2.13-$ $2.29(\mathrm{~m}, 4 \mathrm{H}), 2.35(\mathrm{~s}, 3 \mathrm{H}), 4.06(\mathrm{q}, J=7.1 \mathrm{~Hz}, 2 \mathrm{H})$, $5.07(\mathrm{~s}, 1 \mathrm{H}), 6.25(\mathrm{~s}, 1 \mathrm{H}), 7.08-7.13(\mathrm{~m}, 1 \mathrm{H}), 7.18-7.23$ (m, 2H), 7.28-7.33 (m, 2H). ESI-MS $(m / z) 340(\mathrm{M}+$ $\mathrm{H})^{+}$. Analysis calculated for $\mathrm{C}_{21} \mathrm{H}_{25} \mathrm{NO}_{3}: \mathrm{C}, 74.31 ; \mathrm{H}$, 7.42; N, 4.13. Found: C, 74.27; H, 7.39; N, 4.08.

2.5b Ethyl-1,4,5,6,7,8-hexahydro-2,7,7-trimethyl-5oxo-4-p-tolylquinoline-3-carboxylate (6b): M.p: 260-262 ${ }^{\circ}$. IR $\left(\mathrm{KBr}\right.$ in $\left.\mathrm{cm}^{-1}\right)$ 3287, 3031, 2958, 1644, 1596, 1480, 1334, 1213, 757, ${ }^{1} \mathrm{H}$ NMR $\left(\mathrm{CDCl}_{3}\right.$, $300 \mathrm{MHz}): \delta 0.94(\mathrm{~s}, 3 \mathrm{H}), 1.08(\mathrm{~s}, 3 \mathrm{H}), 1.21(\mathrm{t}, J=$ $7.1 \mathrm{~Hz}, 3 \mathrm{H}), 2.10-2.24(\mathrm{~m}, 4 \mathrm{H}), 2.26(\mathrm{~s}, 3 \mathrm{H}), 2.37$ (s, $3 \mathrm{H}), 4.06(\mathrm{q}, J=7.1 \mathrm{~Hz}, 2 \mathrm{H}), 5.03(\mathrm{~s}, 1 \mathrm{H}), 5.96(\mathrm{~s}$, $1 \mathrm{H}), 7.02(\mathrm{~d}, J=8.0 \mathrm{~Hz}, 2 \mathrm{H}), 7.19(\mathrm{~d}, J=8.0 \mathrm{~Hz}$, $2 \mathrm{H})$. ESI-MS $(m / z) 354(\mathrm{M}+\mathrm{H})^{+}$. Analysis calculated for $\mathrm{C}_{22} \mathrm{H}_{27} \mathrm{NO}_{3}$ : C, 74.76; H, 7.70; N, 3.96. Found: C, 74.74; H, 7.65; N, 3.91.

2.5c Ethyl-1,4,5,6,7,8-hexahydro-4-(4-methoxyphenyl)2,7,7-trimethyl-5-oxoquinoline-3-carboxylate (6c): M.p: $256-257^{\circ} \mathrm{C}$. IR ( $\mathrm{KBr}$ in cm $\left.\mathrm{cm}^{-1}\right) 3281,3199,3080$, 1708, 1607, 1224, 837, ${ }^{1} \mathrm{H}$ NMR $\left(\mathrm{CDCl}_{3} 300 \mathrm{MHz}\right.$,): $\delta 0.94(\mathrm{~s}, 3 \mathrm{H}), 1.07(\mathrm{~s}, 3 \mathrm{H}), 1.21(\mathrm{t}, J=7.2 \mathrm{~Hz}$, $3 \mathrm{H}), 2.09-2.20(\mathrm{~m}, 4 \mathrm{H}), 2.36(\mathrm{~s}, 3 \mathrm{H}), 3.73(\mathrm{~s}, 3 \mathrm{H})$, $4.04(\mathrm{q}, J=7.2 \mathrm{~Hz}, 2 \mathrm{H}), 4.99(\mathrm{~s}, 1 \mathrm{H}), 6.06(\mathrm{~s}, 1 \mathrm{H})$, $6.65(\mathrm{~d}, J=7.3 \mathrm{~Hz}, 2 \mathrm{H}), 7.24(\mathrm{~d}, J=7.3 \mathrm{~Hz}, 2 \mathrm{H})$. ESI-MS $(m / z) 370(\mathbf{M}+\mathbf{H})^{+}$. Analysis calculated

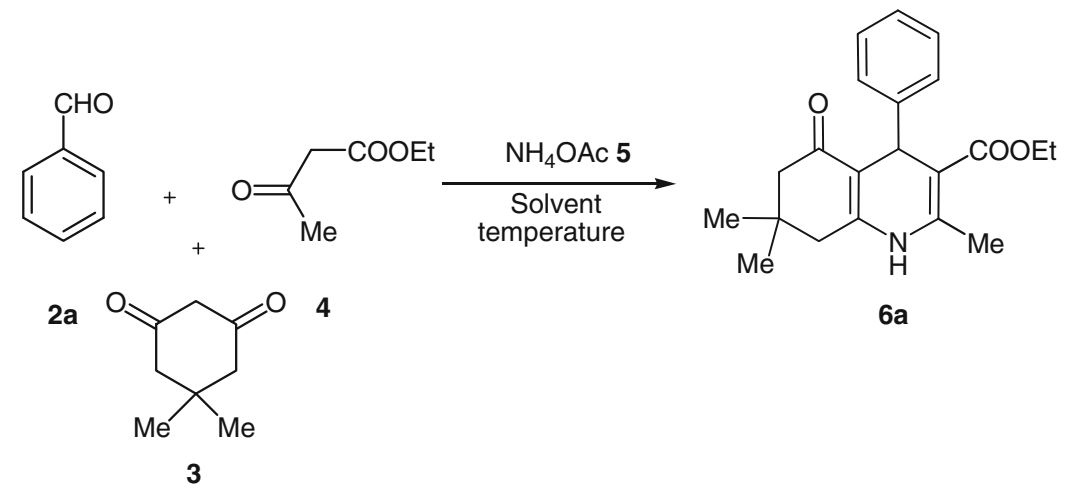

Scheme 1. Optimization studies. 
Table 1. Preparation of deep eutectic solvents 1a-f.

\begin{tabular}{lcc}
\hline Compound A & $\begin{array}{c}\text { Compound B } \\
(\mathbf{a}-\mathbf{f})\end{array}$ & $\begin{array}{c}\text { Ratio A:B } \\
(\mathbf{1 a - f})\end{array}$ \\
\hline Choline chloride & Urea $(\mathbf{a})$ & $1: 2(\mathbf{1 a})$ \\
Choline chloride & 1-Methyl urea $(\mathbf{b})$ & $1: 2(\mathbf{1 b})$ \\
Choline chloride & Thiourea(c) & $1: 2(\mathbf{1 c})$ \\
Choline chloride & Malonic acid $(\mathbf{d})$ & $1: 1(\mathbf{1 d})$ \\
Choline chloride & Phenyl acetic acid $(\mathbf{e})$ & $1: 1(\mathbf{1 e})$ \\
Choline chloride & Glycerin $(\mathbf{f})$ & $1: 1(\mathbf{1 f})$ \\
\hline
\end{tabular}

Table 2. Optimization studies.

\begin{tabular}{lcccc}
\hline $\begin{array}{l}\text { Entry } \\
\text { No. }\end{array}$ & Solvent & $\begin{array}{c}\text { Time } \\
(\mathrm{min})\end{array}$ & $\begin{array}{c}\text { Temp } \\
\left({ }^{\circ} \mathrm{C}\right)\end{array}$ & $\begin{array}{c}\text { Yield } \\
(\%)^{\mathrm{a}, \mathrm{b}}\end{array}$ \\
\hline 1 & $\mathbf{1 a}$ & 20 & 60 & 95.0 \\
2 & $\mathbf{1 b}$ & 20 & 60 & 90.8 \\
3 & $\mathbf{1 c}$ & 20 & 60 & 89.7 \\
4 & $\mathbf{1 d}$ & 20 & 60 & 93.3 \\
5 & $\mathbf{1 e}$ & 20 & 60 & 90.0 \\
6 & $\mathbf{1 f}$ & 20 & 60 & 80.0 \\
7 & $\mathbf{1 a}$ & 60 & 60 & 95.0 \\
8 & $\mathbf{1 a}$ & 20 & 80 & 95.0 \\
9 & $\mathrm{H}{ }_{2} \mathrm{O}$ & 20 & 60 & 73.4 \\
10 & - & 360 & 60 & $65.7^{\mathrm{c}}$ \\
11 & $\mathrm{DMF}$ & 20 & 60 & $52.3^{\mathrm{c}}$ \\
12 & $\mathrm{CH}{ }_{3} \mathrm{CN}$ & 20 & 60 & $38.2^{\mathrm{c}}$ \\
13 & $\mathrm{PhMe}$ & 20 & 60 & $55.3^{\mathrm{c}}$ \\
\hline
\end{tabular}

a $2 \mathbf{a}(1.0 \mathrm{mmol}), \mathbf{3}(1.0 \mathrm{mmol}), 4(1.0 \mathrm{mmol}), 5$ (1.5 mmol), DES $(2.0 \mathrm{~mL})$ at $60^{\circ} \mathrm{C}$ for given time.

${ }^{\mathrm{b}}$ Isolated yields following purification by recrystallization.

${ }^{\mathrm{c}}$ Lower selectivity obtained due to by-product formation

for $\mathrm{C}_{22} \mathrm{H}_{27} \mathrm{NO}_{4}$ : C, 71.54; $\mathrm{H}, 7.31 ; \mathrm{N}, 3.79$; Found: C, 71.59; H, 7.35; N, 3.84.

2.5d Ethyl-1,4,5,6,7,8-hexahydro-4-(4-hydroxyphenyl)2,7,7-trimethyl-5-oxoquinoline-3-carboxylate (6d): M.p: $232-234^{\circ} \mathrm{C}$. IR $\left(\mathrm{KBr}\right.$ in $\left.\mathrm{cm}^{-1}\right) 3285,1690,1617$, 1229, ${ }^{1} \mathrm{H}$ NMR $\left(\mathrm{CDCl}_{3}, 300 \mathrm{MHz}\right): \delta 0.94(\mathrm{~s}, 3 \mathrm{H}), 1.08$ (s, 3H), 1.19 (t, $J=7.2 \mathrm{~Hz}, 3 \mathrm{H}), 2.09-2.19(\mathrm{~m}, 4 \mathrm{H})$, $2.34(\mathrm{~s}, 3 \mathrm{H}), 4.06(\mathrm{q}, J=7.6 \mathrm{~Hz}, 2 \mathrm{H}), 4.72(\mathrm{~s}, 1 \mathrm{H})$, $4.98(\mathrm{~s}, 1 \mathrm{H}), 5.61(\mathrm{~s}, 1 \mathrm{H}), 6.65(\mathrm{~d}, J=8.9 \mathrm{~Hz}, 2 \mathrm{H})$, $7.17(\mathrm{~d}, J=8.4 \mathrm{~Hz}, 2 \mathrm{H})$. ESI-MS $(m / z) 356(\mathrm{M}+$ $\mathrm{H})^{+}$. Analysis calculated for $\mathrm{C}_{21} \mathrm{H}_{25} \mathrm{NO}_{4}$ : C, 70.98; $\mathrm{H}$, 7.04; N, 3.94; Found: C, 70.96; H, 7.02; N, 3.93.

2.5e Ethyl-1,4,5,6,7,8-hexahydro-2,7,7-trimethyl-4(4-nitrophenyl)-5-oxoquinoline-3-carboxylate (6e): M.p: $241-243^{\circ} \mathrm{C}$. IR $\left(\mathrm{KBr}\right.$ in $\left.\mathrm{cm}^{-1}\right) 3290,3150,1711$, 1657, ${ }^{1} \mathrm{H}-\mathrm{NMR}\left(\mathrm{CDCl}_{3} 300 \mathrm{MHz}\right): \delta 0.9(\mathrm{~s}, 3 \mathrm{H}), 1.08$ $(\mathrm{s}, 3 \mathrm{H}), 1.82(\mathrm{t}, J=7.1 \mathrm{~Hz}, 3 \mathrm{H}), 2.16-2.36(\mathrm{~m}, 4 \mathrm{H})$, $2.40(\mathrm{~s}, 3 \mathrm{H}), 4.01(\mathrm{q}, J=7.1 \mathrm{~Hz}, 2 \mathrm{H}), 5.15(\mathrm{~s}, 1 \mathrm{H})$, $6.31(\mathrm{~s}, 1 \mathrm{H}), 7.51(\mathrm{~d}, J=8.2 \mathrm{~Hz}, 2 \mathrm{H}), 8.09(\mathrm{~d}, J=$ $8.3 \mathrm{~Hz}, 2 \mathrm{H})$. ESI-MS $(\mathrm{m} / z) 385(\mathrm{M}+\mathrm{H})^{+}$. Analysis calculated for $\mathrm{C}_{21} \mathrm{H}_{25} \mathrm{~N}_{2} \mathrm{O}_{5} \mathrm{C}, 65.45 ; \mathrm{H}, 6.49 ; \mathrm{N}, 7.27$. Found: C, 65.43; H, 6.49; N, 7.28.

$2.5 f$ Ethyl-4-(2-chlorophenyl)-1,4,5,6,7,8-hexahydro2,7,7-trimethyl-5-oxoquinoline-3-carboxylate (6f): M.p: $208-210^{\circ} \mathrm{C}$. IR $\left(\mathrm{KBr}\right.$ in cm$\left.{ }^{-1}\right) 3062,2955,1720$, 1640, 1610, 1468, 1385, 1228, 1020, 745, ${ }^{1} \mathrm{H}$ NMR $\left(\mathrm{CDCl}_{3}, 300 \mathrm{MHz}\right): \delta 0.95(\mathrm{~s}, 3 \mathrm{H}), 1.07$ (s, 3H), 1.17 $(\mathrm{t}, J=7.1 \mathrm{~Hz}, 3 \mathrm{H}), 2.15-2.23(\mathrm{~m}, 4 \mathrm{H}), 2.30(\mathrm{~s}, 3 \mathrm{H})$, $4.04(\mathrm{q}, J=7.1 \mathrm{~Hz}, 2 \mathrm{H}), 5.38(\mathrm{~s}, 1 \mathrm{H}), 6.11(\mathrm{~s}, 1 \mathrm{H})$, 6,98 (m, 1H), 7.15-7.19 (m, 1H), 7.24-7.26 (m, 1H), 7.41-7.44 (m, 1H). ESI-MS $(m / z) 374(\mathrm{M}+\mathrm{H})^{+}$. Analysis calculated for $\mathrm{C}_{21} \mathrm{H}_{24} \mathrm{ClNO}_{3}$ : C, 67.43; H, 6.47; N, 3.75. Found: C, 67.44; H, 6.48; N, 3.72.

2.5g Ethyl-4-(4-(dimethylamino)phenyl)-1,4,5,6,7,8hexahydro-2,7,7-trimethyl-5-oxoquinoline-3-carboxylate (6i): M.p: $229-231^{\circ} \mathrm{C}$. IR $\left(\mathrm{KBr}\right.$ in $\left.\mathrm{cm}^{-1}\right)$ 3287, 3018, 2964, 1602, 1510, 1377, 1207, 751, ${ }^{1} \mathrm{H}$ NMR $\left(\mathrm{CDCl}_{3}\right.$, $300 \mathrm{MHz}): \delta 0.96(\mathrm{~s}, 3 \mathrm{H}), 1.07(\mathrm{~s}, 3 \mathrm{H}), 1.22(\mathrm{t}, J=$ $7.1 \mathrm{~Hz}, 3 \mathrm{H}), 2.18-2.27$ (m, 4H), 2.35 (s, 3H), 2.86 (s, $6 \mathrm{H}), 4.05(\mathrm{q}, J=7.1 \mathrm{~Hz}, 2 \mathrm{H}), 4.95(\mathrm{~s}, 1 \mathrm{H}), 5.88(\mathrm{~s}$, $1 \mathrm{H}), 6.60(\mathrm{~d}, J=8.6 \mathrm{~Hz}, 2 \mathrm{H}), 7.16(\mathrm{~d}, J=8.6 \mathrm{~Hz}$, $2 \mathrm{H})$. ESI-MS $(m / z) 383(\mathrm{M}+\mathrm{H})^{+}$. Analysis calculated for $\mathrm{C}_{23} \mathrm{H}_{30} \mathrm{~N}_{2} \mathrm{O}_{3}$ : C, 72.22; H, 7.91; N, 7.32. Found: C, 72.18; H, 7.85; N, 7.25.
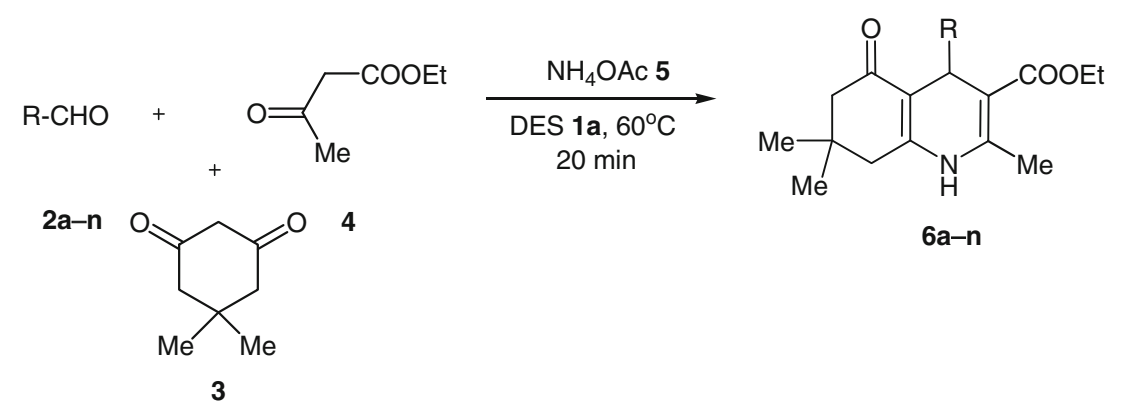

Scheme 2. Synthesis of 1,4-DHP's 6a-n using DES 1a as the solvent. 
Table 3. Synthesis of 1,4-DHPs 6a-n using DES 1a as the solvent.

Entry No. R<smiles>CCOC(=O)C1=C(C)NC2=C(C(=O)CC(C)(C)C2)C1c1ccccc1</smiles><smiles>CCOC(=O)C1=C(C)NC2=C(C(=O)CC(C)(C)C2)C1c1ccc(C)cc1</smiles><smiles>CCOC(=O)C1=C(C)NC2=C(C(=O)CC(C)(C)C2)C1c1ccc(OC)cc1</smiles><smiles>CCOC(=O)C1=C(C)NC2=C(C(=O)CC(C)(C)C2)C1c1ccc(O)cc1</smiles><smiles>CCOC(=O)C1=C(C)NC2=C(C(=O)CC(C)(C)C2)C1c1ccc([N+](=O)[O-])cc1</smiles><smiles>CCOC(=O)C1=C(C)NC2=C(C(=O)CC(C)(C)C2)C1c1ccccc1Cl</smiles><smiles>COc1cc(C=O)ccc1O</smiles>

$2 \mathrm{~g}$<smiles>CCOC(=O)C1=C(C)NC2=C(C(=O)CC(C)(C)C2)C1c1ccc(O)c(OC)c1</smiles> 
Table 3. (continued).

\begin{tabular}{|c|c|c|c|c|c|}
\hline Entry No. & $\mathrm{R}$ & & Product & & Yield $(\%)^{\mathrm{a}, \mathrm{b}}$ \\
\hline 8 & & $2 h$ & & $6 h$ & 73 \\
\hline 9 & $4-(\mathrm{Me})_{2} \mathrm{~N}\left(\mathrm{C}_{6} \mathrm{H}_{4}\right)$ & $2 \mathbf{i}$ & & $6 \mathbf{i}$ & 92 \\
\hline 10 & 4- $\mathrm{Cl}\left(\mathrm{C}_{6} \mathrm{H}_{4}\right)$ & $2 \mathbf{j}$ & & $6 \mathbf{j}$ & 94 \\
\hline 11 & $3-\mathrm{O}_{2} \mathrm{~N}\left(\mathrm{C}_{6} \mathrm{H}_{4}\right)$ & $2 k$ & & $6 \mathbf{k}$ & 93 \\
\hline 12 & $\mathrm{CH}_{3}$ & 21 & & 61 & 83 \\
\hline 13 & $\mathrm{C}_{2} \mathrm{H}_{5}$ & $2 m$ & & $6 m$ & 81 \\
\hline 14 & $\mathrm{n}-\mathrm{C}_{4} \mathrm{H}_{9}$ & $2 n$ & & $6 n$ & 79 \\
\hline
\end{tabular}

a $2 \mathbf{a}-\mathbf{n}(1.0 \mathrm{mmol}), \mathbf{3}(1.0 \mathrm{mmol}), \mathbf{4}(1.0 \mathrm{mmol}), \mathbf{5}(1.5 \mathrm{mmol}), \mathrm{DES} \mathbf{1 a}(2.0 \mathrm{~mL})$ at $60^{\circ} \mathrm{C}$ for $20 \mathrm{~min}$.

${ }^{\mathrm{b}}$ Isolated yields following purification by recrystallization 
2.5h Ethyl-4-(4-chlorophenyl)-1,4,5,6,7,8-hexahydro2,7,7-trimethyl-5-oxoquinoline-3-carboxylate (6j): M.p: $245-246^{\circ} \mathrm{C}$. IR $\left(\mathrm{KBr}\right.$ in $\left.\mathrm{cm}^{-1}\right) 3275,3200,3075$, $2965,1705,1650,1605,{ }^{1} \mathrm{H} \mathrm{NMR}\left(\mathrm{CDCl}_{3}, 300 \mathrm{MHz}\right)$ : $\delta 0.93(\mathrm{~s}, 3 \mathrm{H}), 1.08(\mathrm{~s}, 3 \mathrm{H}), 1.19(\mathrm{t}, J=7.1 \mathrm{~Hz}, 3 \mathrm{H})$, 2.15-2.23 (m, 4H), $2.38(\mathrm{~s}, 3 \mathrm{H}), 4.04(\mathrm{q}, J=7.1 \mathrm{~Hz}$, 2H), $5.02(\mathrm{~s}, 1 \mathrm{H}), 5.90(\mathrm{~s}, 1 \mathrm{H}), 7.15-7.19(\mathrm{~m}, 2 \mathrm{H})$, 7.24-7.26 (m, 2H). ESI-MS $(m / z) 374(\mathrm{M}+\mathrm{H})^{+}$. Analysis calculated for $\mathrm{C}_{21} \mathrm{H}_{24} \mathrm{ClNO}_{3}: \mathrm{C}, 67.46 ; \mathrm{H}$, 6.47; N, 3.75. Found: C, 67.43; H, 6.49; N, 3.73.

2.5i Ethyl-1,4,5,6,7,8-hexahydro-2,7,7-trimethyl-4(3-nitrophenyl)-5-oxoquinoline-3-carboxylate (6k): M.p: $177-178^{\circ} \mathrm{C}$. IR $\left(\mathrm{KBr}\right.$ in cm$\left.{ }^{-1}\right) 3303,2954,1683$, 1610, 1167, 759, ${ }^{1} \mathrm{H}$ NMR $\left(\mathrm{CDCl}_{3}, 300 \mathrm{MHz}\right): \delta 0.89$ (s, 3H), 1.05 (s, 3H), 1.22 (t, $J=7.1 \mathrm{~Hz}, 3 \mathrm{H}), 2.12$ $2.23(\mathrm{~m}, 4 \mathrm{H}), 2.38(\mathrm{~s}, 3 \mathrm{H}) 4.03(\mathrm{q}, J=7.1 \mathrm{~Hz}, 2 \mathrm{H})$, $5.16(\mathrm{~s}, 1 \mathrm{H}), 6.85(\mathrm{~s}, 1 \mathrm{H}), 7.36(\mathrm{t}, J=7.9 \mathrm{~Hz}, 1 \mathrm{H})$, $7.71(\mathrm{~d}, J=7.9 \mathrm{~Hz}, 1 \mathrm{H}), 7.97(\mathrm{~m}, 1 \mathrm{H}), 7.99(\mathrm{~m}, 1 \mathrm{H})$. ESI-MS $(m / z) 385(\mathrm{M}+\mathrm{H})^{+}$. Analysis calculated for $\mathrm{C}_{21} \mathrm{H}_{25} \mathrm{~N}_{2} \mathrm{O}_{5}$ : C, 65.45; $\mathrm{H}, 6.49 ; \mathrm{N}, 7.27$. Found: C, 65.44; H, 6.48; N, 7.26.

2.5j Ethyl-1,4,5,6,7,8-hexahydro-2,4,7,7-tetramethyl5-oxoquinoline-3-carboxylate (6l): M.p: $201-203^{\circ} \mathrm{C}$. IR $\left(\mathrm{KBr}\right.$ in $\left.\mathrm{cm}^{-1}\right)$ 3287, 2947, 1685, 1147, 751, ${ }^{1} \mathrm{H}$ NMR $\left(\mathrm{CDCl}_{3}, 300 \mathrm{MHz}\right): \delta 0.98(\mathrm{~d}, 3 \mathrm{H}), 1.09(\mathrm{~s}, 6 \mathrm{H})$, $1.26-1.31(\mathrm{t}, 3 \mathrm{H}), 2.11-2.32(\mathrm{~m}, 7 \mathrm{H}), 3.91-3.93(\mathrm{q}, 1 \mathrm{H})$, 4.17-4.18(m, 2H), $5.6(\mathrm{~s}, 1 \mathrm{H})$. ESI-MS $(\mathrm{m} / z) 278(\mathrm{M}+$ $\mathrm{H})^{+}$. Analysis calculated for $\mathrm{C}_{16} \mathrm{H}_{23} \mathrm{NO}_{3}: \mathrm{C}, 69.31 ; \mathrm{H}$, 8.30; N, 5.05. Found: C, 69.29; H, 8.29; N, 5.06.

2.5k Ethyl-4-ethyl-1,4,5,6,7,8-hexahydro-2,7,7-trimethyl5-oxoquinoline-3-carboxylate (6m): M.p: 143 $145^{\circ} \mathrm{C}$. IR $\left(\mathrm{KBr}\right.$ in $\left.\mathrm{cm}^{-1}\right)$ 3288, 2954, 1686, 1167, 759, ${ }^{1} \mathrm{H}$ NMR $\left(\mathrm{CDCl}_{3}, 300 \mathrm{MHz}\right): \delta 0.74(\mathrm{t}, J=7.2 \mathrm{~Hz}$ $3 \mathrm{H}), 1.10(\mathrm{~s}, 6 \mathrm{H}), 1.26-1.30(\mathrm{~m}, 5 \mathrm{H}), 2.12-2.34(\mathrm{~m}$, $7 \mathrm{H}), 4.02(\mathrm{t}, J=6.2 \mathrm{~Hz}, 1 \mathrm{H}), 4.16(\mathrm{~m}, 2 \mathrm{H}), 5.58(\mathrm{~s}$, 1H). ESI-MS $(m / z) 292(\mathrm{M}+\mathrm{H})^{+}$. Analysis calculated for $\mathrm{C}_{17} \mathrm{H}_{25} \mathrm{NO}_{3}$ : C, 70.10; H, 8.59; N, 4.89. Found: C, $70.12 ; \mathrm{H}, 8.57 ; \mathrm{N}, 4.87$.

2.51 Ethyl-4-butyl-1,4,5,6,7,8-hexahydro-2,7,7-trimethyl5-oxoquinoline-3-carboxylate (6n): M.p: $165-167^{\circ} \mathrm{C}$. IR $\left(\mathrm{KBr}\right.$ in $\left.\mathrm{cm}^{-1}\right)$ 3290, 3090, 1690, 1197, 751, ${ }^{1} \mathrm{H}$ NMR $\left(\mathrm{CDCl}_{3}, 300 \mathrm{MHz}\right): \delta 0.82(\mathrm{t}, J=7.1 \mathrm{~Hz} 3 \mathrm{H})$, $1.09(\mathrm{~s}, 6 \mathrm{H}), 1.15-1.41(\mathrm{~m}, 9 \mathrm{H}), 2.19-2.30(\mathrm{~m}, 7 \mathrm{H})$, $4.01(\mathrm{t}, J=6.1 \mathrm{~Hz}, 1 \mathrm{H}), 4.17(\mathrm{~m}, 2 \mathrm{H}), 5.77(\mathrm{~s}, 1 \mathrm{H})$. ESI-MS $(m / z) 320(\mathbf{M}+\mathbf{H})^{+}$. Analysis calculated for $\mathrm{C}_{19} \mathrm{H}_{29} \mathrm{NO}_{3}$ : C, 71.47; $\mathrm{H}, 9.09 ; \mathrm{N}, 4.38$. Found: C, 71.45; H, 9.07; N, 4.40.

\section{Results and discussion}

We first set out to prepare a series of DESs as mixtures of $\mathbf{A}$ with amides $\mathbf{B a}-\mathbf{c}$ (ratio 1:2) and $\mathbf{A}$ with carboxylic acids Bd-f (ratio 1:1) (scheme 1, table 1). ${ }^{17}$

We first tested DES 1a-f as solvents for the synthesis of 1,4-DHPs in a one-pot multi-component reaction between 2a-n, 3, 4 and 5. A direct comparison of DES as solvent, with water and under solventless conditions was also carried out. Initial experiments suggested that the reactions initiated in DES 1a-f gave better yields of the desired products at an optimum temperature of $60^{\circ} \mathrm{C}$ as compared to water as solvent and under solventless conditions. Interestingly, 1a proved superior to others, enabling the synthesis of the 1,4-DHPs in excellent yield (95.0\%, entry 1, table 2). Polar and non-polar organic solvents (entry 11-13, table 2) when employed as solvents under similar reaction conditions showed poor conversion to the 1,4-DHPs. These results highlight the potential of DESs 1a-f for promoting synthetically important transformations in comparison to the conventional solvent systems. Easy work-up procedures could be employed to obtain the product for DESs as they could be easily washed away with water suggesting their biocompatible nature.

Encouraged by the above results, we probed the applicability of DES 1a as optimum solvent for synthesis of 1,4-DHPs in scheme 2 . A variety of substituted aldehydes underwent smooth conversion to the desired products. The presence of electron-withdrawing or electron-donating substituents had no influence on the outcome of the reaction with both giving excellent yields. Unsubstituted benzaldehyde (entry 1, table 3) gave the best results followed by electron-withdrawing $-\mathrm{NO}_{2}$ (entry 5, table 3) and $-\mathrm{Cl}$ (entry 6, table 3) groups. The electron-donating -OMe (entry 3, table 3 ) or -Me (entry 2, table 3) were not far behind. Highly substituted aldehydes such as vanillin (entry 7 , table 3 )

Table 4. Recycling of deep eutectic solvent in synthesis of 1,4-DHPs.

\begin{tabular}{lc} 
Recycle of DES & Yield $(\%)^{\mathrm{a}, \mathrm{b}}$ \\
\hline Fresh (non-recycled) & 95.0 \\
First & 92.9 \\
Second & 91.7 \\
Third & 90.3 \\
Fourth & 89.8 \\
Fifth & 87.0
\end{tabular}

a $2 \mathbf{a}(1.0 \mathrm{mmol}), 3(1.0 \mathrm{mmol}), 4(1.0 \mathrm{mmol}), 5(1.5 \mathrm{mmol})$, DES $1 \mathrm{a}(2.0 \mathrm{~mL})$ at $60^{\circ} \mathrm{C}$ for $20 \mathrm{~min}$.

${ }^{\mathrm{b}}$ Isolated yields following purification by recrystallization 
and 5-bromo vanillin (entry 8, table 3 ) also gave good to excellent yields of the 1,4-DHP products. It was further observed that even aliphatic aldehydes gave products in good yields (entry $12-13$, table 3 ). The generality of the protocol is thus exhibited allowing efficient synthesis of differently substituted 1,4-DHPs.

\subsection{Recycle study of DESs}

The ability to recover and recycle the solvent used in the reaction is an important aspect of green chemistry. Therefore in the present study, we also evaluated the recyclability and reusability of this DES (table 4).

\section{Conclusion}

In summary, a very simple approach towards onepot multi-component synthesis of 1,4-dihydropyridine derivatives was developed using biocompatible DESs. The DES gave excellent recyclability without loss of activity up to 5 cycles, hence providing a greener alternative. A milder and highly efficient protocol was developed which can easily replace existing methods.

\section{References}

1. Bossert F, Meyer H and Wehinger E 1981 Angew. Chem. Int. Ed. Engl. 20762

2. Mannhold R, Jablonka B, Voigdt W, Schoenafinger K and Schrava K 1992 Eur. J. Med. Chem. 27229

3. Reid G L, Meredith P A and Pasanisi F 1985 J. Cardiovasc. Pharmacol. 7 S18

4. Shan R, Velazquez C and Knaus E 2004 J. Med. Chem. 47254

5. Kawase M, Shah A, Gaveriya H, Motohashi N, Sakagami H, Varga A and Molnar J 2002 Bioorg. Med. Chem. 101051

6. a) Janis R A and Triggle D J 1983 J. Med. Chem. 26 775;

b) Loev B, Goodman M M, Snader K M, Tedeschi R and Macko E 1974 J. Med. Chem. 17956
7. a) Tietze L F 1996 Chem. Rev. 96 115; b) Tietze L F, Brasche G and Gericke K M 2006 Domino reactions in organic synthesis, Weinheim: Wiley-VCH

8. Love B and Sander K M 1965 J. Org. Chem. 301914

9. Tu S-J, Yu C-X, Liu X-H, Yao C-S, Liu F and Gao Y 2002 Chin. J. Struct. Chem. 2199

10. Wang L-M, Sheng J, Zhang L, Han J-W, Fan Z-Y, Tian $\mathrm{H}$ and Qian C-T 2005 Tetrahedron 611539

11. Ji S J, Jiang Z Q, Lu J and Loh T P 2004 Synlett 831

12. a) Welton T 1999 Chem. Rev. 99 2071; b) Ranke J, Stolte S, Stormann R, Arning J and Jastorff B 2007 Chem. Rev. 107 2183; c) Jastorff B, Stormann R, Ranke J, Molter K, Stock F, Oberheitmann B, Hoffmann W, Hoffmann J, Nuhter M, Ondruschka B and Filser J 2003 Green Chem. 5 136; d) Stolte S, Arning J, Bottin-Weber U, Matzke M, Stock F, Thiele K, Uerdingen M, Welz-Biermann U, Jastorff B and Ranke J 2006 Green Chem. 8 621; e) Anastas P T 2010 Green solvents: ionic liquids, P Wasserscheid and A Stark (eds) Weinheim: WileyVCH, vol. 6

13. Park S and Kazlauskas R J 2003 Curr. Opin. Struct. Biol. 14432

14. Gericke M, Fardim P and Heinze P 2012 Molecules 17 7458

15. a) Aparicio S, Atilhan $M$ and Karadas F 2010 Ind. Eng. Chem. Res. 49 9580; b) Seddon K R, Stark A and Torres M J 2000 Pure Appl. Chem. 72 2275; c) Ab Rani M A, Brant A, Crowhurst L, Dolan A, Lui M, Hassan N H, Hallett J P, Hunt P A Niedermeyer H and PerezArlandis J M 2011 Phys. Chem. Chem. Phys. 13 16831; d) Gallo V, Mastrorilli P, Nobile C F, Romanazzi G and Suranna G P 2002 J. Chem. Soc. Dalton 23 4339; e) Lee S, Ha S, Lee S and Koo Y M 2006 Biotechnol. Lett. 28 1335

16. Shamsuri A A and Abdullah D K 2010 MAKARA SAINS. 14101

17. a) Abbot A, Capper G, Davies D, Munro H, Rasheed $\mathrm{R}$ and Tambyrajah V 2001 Chem. Commun. 2010; b) Abbott A, Capper G, Davies D, Rasheed R and Tambyrajah V 2003 Chem. Commun. 70; c) Abbott A, Capper G, Davies D and Rasheed R 2004 J. Am. Chem. Soc. 1269142

18. Gorke J, Srienc F and Kazlauskas R 2008 Chem. Commun. 1235

19. Abbott A, Cullis P, Gibson M, Harris R and Raven E 2007 Green Chem. 9868

20. Jain N, Kumar A and Chauhan S 2005 Tetrahedron 61 1015 